J. Egypt. Soc. Parasitol. (JESP), 51(1), 2021: 127 - 138

(Online: 2090-2549)

\title{
FLOW CYTOMETRIC AND MOLECULAR ANALYSIS OF POSSIBLE PRO- TOZOAL CONTAMINATION OF DRINKING WATER IN TANTA, EGYPT
}

\section{By}

DALIA A. ELMEHY ${ }^{1 *}$, HOWAIDA I. H. ISMAIL ${ }^{1}$, AMIRA A. ALFATTAH ${ }^{1}$, KHOLOUD A. ELNOUBY ${ }^{1}$, SAHAR M. HAZZAA ${ }^{2}$ and AYMAN A. EL-BADRY ${ }^{3}$

Department of Medical Parasitology ${ }^{1}$, and Department of Clinical Pathology ${ }^{2}$, Faculty of Medicine, Tanta University ${ }^{1,2}$, Tanta, and Department of Medical Parasitology, Faculty of Medicine, Cairo University ${ }^{3}$, Cairo, Egypt

( ${ }^{*}$ Correspondence: dalia.elmehy@med.tanta.edu.eg

- ORCID: https://orcid.org/0000-0001-5590-3424)

\section{Abstract}

Protozoal water contamination is an alarming cause of countless waterborne outbreaks. The most eminent causal protozoa are Cryptosporidium and Giardia species (spp.) as they can endure aquatic environment even with chlorine disinfectants. The currently used traditional techniques cannot permit an easy detection of the waterborne protozoa concerning their count, viability, and pathogenicity. The present work detected protozoal contamination of the drinking water in Egypt with the determination of their load, viability and potential pathogenicity. Four techniques were compared including conventional staining techniques, immunofluorescence (IF) staining, flow cytometry (FC) and molecular study. Also, viability was assessed by conventional trypan blue stain and nucleic acid stain. Along a year, 64 water samples were collected and concentrated from water tanks and tap water of different districts, significant differences $(\mathrm{P}<0.001)$ was between the different techniques in each season regarding the detection of Giardia cysts and Cryptosporidium oocysts. Number of total positive samples was significantly higher in tank water than tap water $(\mathrm{P}<0.001)$ especially at summer. Flow cytometry and nested polymerase chain reaction (nPCR) proved to be much more sensitive than IF assay, and conventional staining techniques. Regarding viability, nucleic acid stain was more sensitive than trypan blue stain $(\mathrm{P}<0.001)$. Also, C. parvum predominate other Cryptosporidium genotypes.

Keywords: Cryptosporidium, Giardia, Immunofluorescence staining, Flow cytometry, water, nested PCR.

\section{Introduction}

Unfortunately, safe water, free from pathogens and other contaminants, is not actually obtainable to many of the world population. Being closely associated with human health makes this problem a major public health concern even for the developed countries (McKee and Cruz, 2021). The most recorded protozoal waterborne disease outbreaks (WBDO) were mainly attributed to Cryptosporidium spp. and Giardia spp. They cause diarrheal diseases in healthy individuals and intractable life-threatening illness in immunocompromised patients, annually accounted for more than 22 million deaths (Rosado et al, 2017). In fact, water has a significant risk factor for their transmission as they can survive for several months in aquatic environment and they are resistant to most disinfectants specially chlorination.

Also, they have a low infectious dose, harbbored by many animals (Zahedi et al, 2021).
The detection of Cryptosporidium spp., and Giardia spp. in water using the conventional methods and light microscope was a tedious effort and time-consuming, which required a certain level of experience, due to high contamination intensity. So, more sensitive techniques were needed to avoid sudden protozoal WBDO (Hassan et al, 2021). Flow cytometry is a highly sensitive and specific technique that can detect low levels of waterborne protozoa and identify their count depending on their size and internal complexity (Silva and Sabogal-Paz, 2020). Nested PCR for genotypes detection of protozoa emerged as powerful source tracking method for public health risk assessment, and pathogenicity determination (Fan et al, 2021).

Most genotyping studies of Cryptosporidium spp., and Giardia spp. on drinking water were conducted in the developed countries whereas, in the developing ones, there were only limited studies (Feng et al, 2011; 
Yin et al, 2021).

This study aimed to assess the currently available advanced techniques in detecting the possible protozoal contamination of the drinking water at Tanta city the capital of Gharbia Governorate (Nile Delta) and thus, identification of their load, viability and potential pathogenicity

\section{Materials and Methods}

This descriptive, analytical study was conducted on drinking water samples from different districts of Tanta City and the nearby rural areas over one year.

Sample collection: 64 water samples (5-10 liters) were collected in sterile containers, 32 samples (8/season) were collected from water tanks, and other 32 samples (8/season) were collected from tap water. Data regarding site, date, and source of collection were recorded on each sample container. Boiled distilled water was used as a negative control for FC analysis. Source water and filtered tap one were excluded.

Sample processing: a drop of water was directly examined under light microscope using 10x, 40x \& 100xobjectives. Then, all samples were filtered through Whatman cellulose nitrate membrane filters $<2$ microns using pumped stainless-steel filtration unit and membrane filters were eluted with PBS (Silva and Sabogal-Paz, 2020). The samples were centrifuged at $4000 \mathrm{rpm}$ for $15 \mathrm{~min}$., the sediment pellets $(1-5 \mathrm{ml})$ were collected in aliquots for each sample and preserved in potassium dichromate $(2.5 \%)$. The centrifugation force resulted in the recovery of $\mathrm{Gi}$ ardia cysts and Cryptosporidium oocysts (Fontaine and Guillot, 2003). Each sediment pellet was examined by conventional staining, IF staining, FC and molecular study. Viability was assessed using standard trypan blue stain and 4'6-diamidino-2-phenylindole (DAPI) nucleic acid stain.

Conventional stains as the Lugol's iodine, Modified Zeihl-Neelsen (MZN) and Trypan blue (viability stain) were used (Silva and Sabogal-Paz, 2020). Immunofluorescence staining using a specific kit (A100DFK. Aq-
ua-Glo $^{\text {TM }}$ G/C. Direct, Dual Fluorochrome (FL/Cy3), Comprehensive kit Fluorescein \& Cy3-labeled Monoclonal Antibody Reagent, Waterborne Inc., New Orleans, LA) was done. Aqua-Glo ${ }^{\mathrm{TM}} \mathrm{G} / \mathrm{C}$ was approved by Environmental Protection Agency (EPA) for use in methods $1622 \& 1623$ for simultaneous direct immunofluorescence detection of Giardia \& Cryptosporidium (oo) cysts and their viability via DAPI nucleic acid staining by fluorescent microscope (Yoder et al, 2008). Protozoal contamination intensity was determined by conventional \& IF staining techniques. Live and dead parasites were calculated/100 $\mu 1$ using high-power lens. Contamination intensity score was: mild; $<10$ (oo) cysts $/ 100 \mu 1$, moderate; $10-20$ (oo)cysts/ $100 \mu 1$, and severe; $>20$ (oo)cysts/ $100 \mu 1$.

Detection of Giardia and Cryptosporidium (oo) cysts in water samples was done by flow cytometric analysis using the previously kit (Vesey et al, 1994). 100 $\mu 1$ water samples were analyzed on a Flow cytometry Activated Cell Sorter (FACS) Calibur flow cytometer Becton Dickinson equipped with an argonion laser operating at $488 \mathrm{~nm}$. FACS was calibrated using calibrated beads (Becton Dickinson, Mississauga, Ontario, Canada) and samples were analyzed using biparametric histograms. A region was set around the particles found in each sample called the gated region from which different signals were used to sort (oo) cysts from the debris. The signals used for sorting were forward angle light scattering (FSC) and side-angle light scattering (SSC). Forward light scatter was responsible for size determination, and right-angle light scatter to detect internal complexity. Signals were based on fluorescence detected by special detectors for fluorescent stains calibrated to detect each stain by using emission wavelength. Flow cytometer expressed results as a percent of the total particles in water sample introduced to device (events) on the histograms' screen. Number of events and percent of gated events in the quadrant of interest determined the contamination intensity according to the follow- 
ing score system: mild; $1-30 \% / 100 \mu 1$, moderate; $31-70 \% / 100 \mu 1$, and severe; $71-100 \%$ / $100 \mu 1$.

Molecular analysis (Fan et al, 2021): Water samples were kept at $-20^{\circ} \mathrm{C}$ to be assessed by nPCR targeting Triose phosphate isomerase (TPI) gene of Giardia spp., \& $\mathrm{Cr}$ yptosporidium outer wall protein (COWP) gene of Cryptosporidium spp. Positive samples were subjected to restriction enzyme length polymorphism (RFLP) to identify its' genotype. The nPCR detected Giardia TPI gene via two successive reactions in collaboration with two primers: AL3543: 5'- AAAT IATGCCTGCTCGTCG-'3 \& reverse primer AL3546: 5'- CAAACCTTITCCGCAAACC -' 3 for primary reaction to amplify $605 \mathrm{bp}$ DNA and a fragment of 530bp for secondary one using AL3544: 5'- CCCTTCATCGGIG GTAACTT-' 3 and reverse primer AL3545: 5'- GTGGCCACCACICCCGTGCC-'3. Mixed reaction consisted of $1 \mu \mathrm{l}$ of each primer (200nM), $5 \mu \mathrm{l}$ of template DNA, and $12.5 \mu \mathrm{l}$ of Dream Taq Green PCR Master Mix (Product No. K1081: Thermo-Scientific, USA) and molecular grade water to achieve $25 \mu 1$ $\& 2 \mu 1$ of secondary reaction. Cycling conditions were done and the annealing temperature was $45^{\circ} \mathrm{C}$ for both primary and secondary PCR-assays. Amplified products were visualized with $1.5 \%$ agarose gel electrophoresis after ethidium bromide staining. Extraction of COWP gene DNA from water samples was done using Favor-Prep ${ }^{\mathrm{TM}}$ stool DNA isolation Mini Kit (Cat. No. FASTI 001, Favorgen Biotech corporation ping-Tung 908, Taiwan). PCR amplified the COWP gene using primers BCOWPF \& BCOWPR for E-PCR \& Cry-15 and Cry-9 for nPCR.

Reaction master mixture ( $\mathrm{Rx})$ for each PCR set was prepared as one reaction, in a volume of $25 \mu 1$ and multiplied by the samples number. Tube was inserted in the thermal cycler, and thermal profile was adjusted as follow; Initial denaturation at $95^{\circ} \mathrm{C}$ for 5min., and then 35 cycles of amplification consisted of denaturation at $94^{\circ} \mathrm{C}$ for $30 \mathrm{sec}-$ onds, annealing at $55^{\circ} \mathrm{C}$ for 30 seconds and extension at $72^{\circ} \mathrm{C}$ for 30 seconds. Final elongation was done for $10 \mathrm{~min}$ at $72^{\circ} \mathrm{C}$. Secondary PCR reaction was done using the primary reaction amplified product as a template. The same reaction conditions were applied. Amplified samples were run in parallel on $2 \%$ agarose gel using gel electrophoresis and visualized on a UV transilluminator to confirm the amplified PCR (Ursini et al, 2020). After the manufacturer's instructions, following protocol was done to digest nPCR products positive samples for Cryptosporidium after amplification (Aghamolaie et al, 2016). Components for each reaction included $10 \mu \mathrm{l}$ nPCR product (Target DNA), $17 \mu 1$ Nucleasefree water, $2 \mu$ l Green buffer, $1 \mu l$ RsaI Enzyme and $30 \mu l$ to achieve the required total volume. Gentle mixing was done, spinning down for few seconds \& incubation at $37^{\circ} \mathrm{C}$ for $5 \mathrm{~min}$. followed by Cryptosporidium genotypes detection using Agarose Gel Electrophoresis and UV Light Transillumination. The PCR marker and volumes of $6 \mu \mathrm{l}$ of amplified product, after digestion, were slowly loaded into the sample wells. Electrophoresis was performed as given before but the power supply was programmed to give 75 volts for $70 \mathrm{~min}$. Band detection in Agarose Gel was done using UV Transillumination. Cryptosporidium genotypes (Gel interpretation) were done by using restriction enzyme Rsa I, digestion of $\mathrm{nPCR}$ product targeting COWP gene. PCR-RFLP showed the 2 genotypes. Sample was C. parvum if Rsa I digestion gave 4 bands, namely 34bp, 106bp, 125bp \& 285 base pair (bp), and C. hominis if Rsa I digestion gave 3 bands namely 34bp, 106bp \& 410bp.

Statistical analysis: Data were analyzed by SPSS V.16 using chi-square test for comparison between groups. $\mathrm{P}<0.05$ was significant.

\section{Results}

The preliminary examination by direct smear was negative for all samples. Prevalence of Giardia cysts using iodine stain was $15.62 \%$, IF staining was $29.69 \%$, FC was $73.43 \%$, and nPCR was $57.8 \%$ with signifi- 
cant difference $(\mathrm{P}<0.001)$. Positive samples detected in the four seasons were significant ( $\mathrm{P}<0.05)$, with the highest one during summer. Overall prevalence of Cryptosporidium oocysts by using MZN stain was $20.31 \%$, IF staining was $39.06 \%$, FC was $81.25 \%$, and nPCR was $62.5 \%$ with significant difference $(\mathrm{P}<0.001)$. The highest prevalence was during summer with significant difference $(\mathrm{P}$ $<0.05)$.

The nPCR was considered the gold standard method to detect the prevalence and seasonal variations of Giardia cysts and Cryptosporidium oocysts. The relative sensitivity of iodine stain, IF staining and FC in detecting Giardia cysts was $77.7 \%, 79.9 \%$, \& $95.5 \%$, respectively, and specificity was $80 \%, 82.8 \%$ \& $92.9 \%$, respectively.

Total positive samples of Giardia cysts was significantly higher in the tank than in tap water $(\mathrm{P}<0.05)$, but positive samples didn't show a significant difference between both sources seasonally $(\mathrm{P}>0.05)$. Seasonal variation showed the highest number of positive tank and tap water samples with significant difference $(\mathrm{P}<0.05)$.

Comparing different methods in detecting Giardia cysts contamination intensity in tank water in different season, the FC detected the highest positive samples $(81.25 \%)$ followed by nPCR $(68.75 \%)$, with significancy $(\mathrm{P}<0.05)$. Also, the largest number of positive samples were detected during summer $(87.5 \%)$ but without significant results ( $\mathrm{P}$ $>0.05$ ). Regarding detection of Giardia cysts intensity in tap water using different techniques during different season, $\mathrm{FC}$ detected the highest positive samples $(65.62 \%)$ with significant difference $(\mathrm{P}<0.001)$. The highest positive samples for Giardia cysts in tap water were detected during summer but without significant difference $(\mathrm{P}>0.05)$.

Regarding the viability of Giardia cysts in both tank and tap water, results of trypan blue and DAPI stains were significant (P $<0.05$ ) with highest number of viable Giardia cysts during summer. Also, regarding of Giardia cysts intensity contamination, FC was powerful in detection of severe contamination in tank and tap water $(\mathrm{P}<0.05)$.

Considering nPCR a gold standard method, the specificity and sensitivity of Cryptosporidium oocysts detection in all tank and tap water samples using MZN, IF, FC were compared to its' results. The above techniques' sensitivity was $80 \%, 81 \%$, \& $96.2 \%$ respectively, while specificity was $78.9 \%, 83.3 \%$, \& $93.5 \%$ respectively. Total number of positive Cryptosporidium oocysts samples was significantly higher $(\mathrm{P}<0.05)$ in tank water $(37.5 \%)$ than tap water $(25 \%)$. The highest number of total positive samples of tank and tap water was during summer with significant results $(\mathrm{P}<0.05)$. Flow cytometry showed the highest Cryptosporidium oocysts positive samples all tank and tap water, followed by nPCR, with significant differences $(\mathrm{P}<0.05)$. The largest Cryptosporidium oocysts positive samples was by nPCR during summer but without significant differences $(\mathrm{P}>0.05)$.

Regarding the viability of the Cryptosporidium oocysts in both tank and tap water, trypan blue and DAPI stains results were significant $(\mathrm{P}<0.05)$ with the highest Cryptosporidium viable oocysts was during summer. Concerning intensity of Cryptosporidium oocysts contamination, IF stain detected more contamination than iodine stain in tank \& tap water $(\mathrm{P}<0.05)$. FC was powerful in detection of severe contamination in tank and tap water $(\mathrm{P}<0.05)$. The nPCR detected 40/ 64 positive Cryptosporidium samples. RFLP products showed $C$. parvum positive samples in $55 \%$ followed by $C$. hominis in $12.5 \%$, while both genotypes were detected in $25 \%$ of positive samples. Unclassified genotypes were distinguished in $7.5 \%$ of positive samples with significant difference $(\mathrm{P}<0.05)$.

Details were illustrated in figures $(1,2,3$, $4,5,6,7, \& 8)$.

\section{Discussion}

Water pollution is a major global issue which requires regular and continuous evaluation and management. Unsafe water ranks the third among the twenty leading risk fac- 
tors for health burden as it provides a favorable environment for many organisms to thrive. This problem has been a major concern of many countries especially in Egypt (Gad et al, 2020). Most of the Egyptian governorates depend on surface water as the main drinking source. The most common source of protozoal contamination of water sources in Egypt is the fecal source. This results from the improper disposal of sewage which is usually discarded in seas, rivers, lakes, and canals (Abd El-Latif et al, 2020).

The present study reported a significant difference in the number of Giardia cysts and Cryptosporidium oocysts positive samples detected using different diagnostic techniques in each season as well as in the total number of positive samples detected throughout the year. These results could be attributed to the global warming which stimulates parasite growth or to the use of highly sensitive techniques for testing. It also could be related to where the source of samples, as Delta is highly infiltrated with farms with extensive animal breeding activities. Lastly, it could be caused by the high resistance of these parasites to the usually used water disinfectants as chlorine attributed to their strong (oo)cyst walls (Zahedi et $a l, 2021)$. Results of this study coincide with some previous similar studies involving drinking water sources of Egypt, as the study conducted in Nile Delta villages over 35 years ago by Khairy et al. (1982) which reported a high prevalence of Giardia cysts in on both Zir water and tap water (36\%) each. Furthermore, a project that was conducted in Nile Delta governorates reported similar results regarding the prevalence of such protozoa (El-Kowrany et al, 2016). This indicates that nothing was done either to monitor the prevalence of such protozoa in water nor to treat the water supplies. However, a study conducted in Dakhahlia governorate reported less contamination of the drinking water with Cryptosporidium and Giardia (oo) cysts $3.1 \%$ and $2.1 \%$ respectively (El Shazly et al, 2007). This could be attributed to the use of a less sensitive technique for detection.

More studies all over the world assessed parasitic contamination of the drinking water, for example, Briancesco and Bonadonna, (2005) performed a study in Italy and reported that Cryptosporidium and Giardia are present in untreated sewage and surface source water, and they disappear after water treatment. Moreover, in the UK, Cryptosporidium was detected in drinking water in $100 \%$ of the samples using nPCR and RFLP (Nichols et al, 2003). Also, Feng et al. (2011) confirmed the same results. On the contrary, in the USA low parasites prevalence was reported by LeChevallier et al. (1991) as Giardia was only found in $17 \%$ and Cryptosporidium in only $27 \%$ of filtered water samples.

In the current study, revealed that the total protozoal positive samples were significantly higher in tank water than tap water. This agreed with Baldursson and Karanis (2011) and (Rosado-García et al. (2017). In the present study, the highest prevalence of Cryptosporidium and Giardia (oo) cysts was during summer. This agreed with Antonios et al. (2010); El-Kowrany et al. (2016); Brankston et al. (2018) and Xiao et al. (2018), who reported that Cryptosporidium contamination positive degree correlated with warm weather. But, Ibrahim et al. (2020) recorded Giardia in river water in rainy seasons.

In the current study, Lugol's iodine identified Giardia cysts. Also, MZN stain was proven to be fast, simple, sensitive and the most effective stain for detection of Cryptosporidium oocysts. This was confirmed by many studies performed all over the world which compared it to many other stains (Adeyemo et al, 2018; Hassan et al, 2021). Immunofluorescence assay was proved to be an efficient technique especially with low levels of (oo)cysts as it represents sensitive, specific, and time saving than the traditional stains (Quintero et al, 2002). However, its use was limited because it required a fluorescent microscope, expensive reagents, and 
didn't distinguish viable and dead organisms (Alles et al, 1995). Also, Aghamolaie et al. (2016) found that MZN staining gave good accuracy for Cryptosporidium diagnosis due to its low costs and inaccessibility of other techniques in all laboratories.

The FC facilitated detection and determination of the intensity of contamination by both protozoa and proved to be more sensitive than the conventional and IF staining. This agreed with Vesey et al. (1993) who applied FC as a routine assessment for detection of Cryptosporidium and Giardia (oo) cysts in water samples. They had proved that FC detected more positive samples than microscopy. Although FC was proved to be a simple rapid and sensitive technique, its use is limited because it is much more expensive than the conventional methods. Moreover, it does not detect any simultaneously present protozoa present in the collected samples as it is confined to the used specific monoclonal antibody (Vesey et al, 1994). Moreover, the FC overestimated the intensity of contamination as it could not distinguish the algae that have the same size of protozoa and show the same fluoresce too. However, the percentage of these particles was often negligible, their fluorescence intensity usually very low, and was not incriminate to have a significant effect on results (Valdez et al, 1997; Silva and Sabogal-Paz, 2020).

In the current study, nPCR was considered the highly sensitive gold standard method, and detected the DNA of Giardia and Cryptosporidium spp. This agreed with Nichols et al. (2003) who reported its rapid and less liable for post-amplification contamination, with more positive samples than conventional and IF stains ones. Also, Fontaine and Guillot (2003) compared between IF stain and PCR, and found that PCR was more valuable. Moreover, nPCR revealed fewer positive samples than FC regards Cryptosporidium and Giardia (oo) cysts. This agreed with Valdez et al. (1997), where only $25 \%$ of $\mathrm{FC}$ the positive samples were confirmed by the PCR. No doubt, FC not only detected viable (oo) cysts but also detectd empty ones (ghosts) which led to a higher false result.

In the present study, $C$. parvum and $C$. hominis were the commonest genotypes affecting humans. RFLP showed that C. parvum was the dominant species due to high prevalence of bovine cryptosporidiosis in the Nile Delta. This agreed with McLauchlin et al. (2000) who reported that C. parvum was the major cause of cryptosporidiosis affected bovines and immunocompromised human patients in the UK. But, only $12.5 \%$ of Cryptosporidium positive samples were due to $C$. hominis. Also, others reported a similar prevalence of the $C$. hominis genotype in water samples and human stool samples (Chalmers et al, 2010), as a delicate and less resistant species with relatively lower incidence (Chalmers et al, 2011).

In the present study, also the $7.5 \%$ unclassified Cryptosporidium genotypes in the positive samples, may be $C$. baileyi and $C$. meleagridis, mainly birds' parasites contaminated uncovered water tanks (Gibson et al. 2011). C. baileyi genotype was found in one sample by either of Nichols et al. (2006) and Cardozo et al. (2008).

In the present study, the high percentage of viable Cryptosporidium and Giardia (oo) cysts in drinking chlorinated water. Nakada et al. (2019) reported Giardia cyst resisted chlorine. The DAPI stain proved to be significantly superior to the trypan blue stain in detecting the viability of both Cryptosporid ium and Giardia (oo) cysts because only viable nuclei uptake the DAPI. This agreed with Rousseau et al. (2018) and Burgt et al. (2018) who used fluorochrome DAPI to predict viability of Cryptosporidium oocysts.

Since DAPI staining technique only required one step for preparation, it was considered more efficient than the tedious trypan blue stain which consumes much time to detect the percentage of live parasites in the sample under a conventional microscope. To enhance the results, both DAPI stain and FC could be used together to detect the percent- 
age of the viable (oo) cysts (Medema et al, 1998 and Sammarro et al, 2020. Unfortunately, this was not accessible for this study as DAPI had a different wavelength than the used fluorochromes (FITC and Cy3) which was not provided in the $\mathrm{FC}$ device.

\section{Conclusion}

Regarding detection of Cryptosporidium and Giardia (oo) cysts in water, the conventional microscope is still the cheapest available technique but with lower sensitivity compared to the recent techniques as FC. Also, nPCR proved to be more sensitive than conventional staining techniques as it detects the parasite DNA and distinguished different genotypes. However, being expensive made it not feasible to be used as a routine assessment. It was recommended to design national standards for detection of all parasitic contaminations of water.

\section{Acknowledgement}

This study was kindly supported by Tanta University, the Project No. TU.01.13.04.

\section{References}

Abd El-Latif, NF, El-Taweel, HA, Gaballah, A, Salem, AI, Abd El-Malek, AHM, 2020: Molecular characterization of Giardia intestinalis detected in humans and water samples in Egypt. Acta Parasitol. 182:1-8.

Adeyemo, FE, Singh, G, Reddy, P, Stenström, TA, 2018: Methods for the detection of Cryptosporidium and Giardia from microscopy to nucleic acid-based tools in clinical and environmental regimes. Acta Trop. 184:15-28.

Aghamolaie, S, Rostami, A, Fallahi, S, Biderouni, FT, Haghighi, A, et al, 2016: Evaluation of modified Ziehl-Neelsen, direct fluorescentantibody and PCR assay for detection of Cryptosporidium spp. in children fecal specimens. J. Parasit. dis. 40, 3:958-63.

Alles, AJ, Waldron, MA, Sierra, LS, Mattia, AR 1995: Prospective comparison of direct immunofluorescence \& conventional staining methods for detection of Giardia and Cryptosporidium spp. in human fecal specimens. J. Clin. Microbiol. 33, 6:1632-4.

Antonios, SN, Tolba, OA, Othman, AA, Saad, M, 2010: A preliminary study on the prevalence of parasitic infections in immunocompromised children. J. Egypt. Soc. Parasitol. 40, 3:617-30.
Baldursson, S, Karanis, P, 2011: Waterborne transmission of protozoan parasites: Review of worldwide outbreaks-an update 2004-2010. Water Res. 45, 20: 6603-6614.

Brankston, G, Boughen, C, Ng, V, Fisman, D N, Sargeant, JM, et al, 2018: Assessing the impact of environmental exposures and Cryptosporidium infection in cattle on human incidence of cryptosporidiosis in Southwestern Ontario, Canada. PLoS One 13, 4:e196573.

Briancesco, R, Bonadonna, L, 2005: An Italian study on Cryptosporidium and Giardia in wastewater, fresh water and treated water. Environ. Monit. Assess. 104, 1:445-457.

Burgt, NHV, Auer, A, Zintl, A, 2018: Comparison of in vitro viability methods for Cryptosporidium oocysts. Exp. Parasitol. 187, 30-36.

Cardozo, Sergian, V, Walter, L, Teixeira, F, Lopes, CW, 2008: Evaluation of different diagnostic techniques for oocysts of Cryptosporidium baileyi in broiler chickens (Gallus gallus domesticus) fecal samples. Rev. Bras. Parasitol. Vet. 17:351-3.

Chalmers, RM, Robinson, G, Elwin, K, Hadfield, SJ, Thomas, E, et al, 2010: Detection of Cryptosporidium species and sources of contamination with Cryptosporidium hominis during a waterborne outbreak in north-west Wales. J. Water Hlth. 8, 2: 311-25.

Chalmers, RM, Smith, R, Elwin, K, CliftonHadley, FA, Giles, M, 2011: Epidemiology of anthroponotic and zoonotic human cryptosporidiosis in England \& Wales, 2004-2006. Epidemiol. Infect. 139, 5: 700-12.

El-Kowrany, SI, El-Zamarany, EA, El-Nouby, KA, El-Mehy, DA, Ali, EAA, et al, 2016: Water pollution in the Middle Nile Delta, Egypt: An environmental study. J. Adv. Res. 7, 5:78194.

Elshazly, AM, Elsheikha, HM, Soltan, DM, Mohammad, KA, Morsy, TA, 2007: Protozoal pollution of surface water sources in Dakahlia Governorate, Egypt. J. Egypt. Soc. Parasitol. 37, 1:51-64.

Fan, Y, Wang, X, Yang, R, Zhao, W, Li, N, et al, 2021: Molecular characterization of the waterborne pathogens Cryptosporidium spp., Giardia duodenalis, Enterocytozoon bieneusi, Cyclospora cayetanensis and Eimeria spp. in wastewater and sewage in Guangzhou, China. Parasit. Vectors 14, 1:1-10.

Feng, Y, Zhao, X, Chen, J, Jin, W, Zhou, X, et al, 2011: Occurrence, source, and human infect- 
ion potential of Cryptosporidium and Giardia spp. in source and tap water in Shanghai, China. Appl. Environ. Microbiol. 77, 11:3609-16.

Fontaine, $M$ and Guillot, E, 2003: An immunomagnetic separation-real-time PCR method for quantification of Cryptosporidium parvum in water samples. J. Microbiol. Meth. 54, 1:29-36.

Gad, M, Elsayed, S, Moghanm, FS, Almarshadi, MH, Alshammari, AS, et al, 2020: Combining water quality indices and multivariate modeling to assess surface water quality in the Northern Nile Delta, Egypt. Water 12, 8:21-42.

Gibson-Kueh, S, Yang, R, Thuy, NTN, Jones, JB, Nicholls, PK et al, 2011: The molecular characterization of an Eimeria and Cryptosporidium detected in Asian seabass (Lates calcarifer) cultured in Vietnam. Vet. Parasitol. 181, 4:91-6.

Hassan, EM, Örmeci, B, DeRosa, MC, Dixon, BR, Sattar, SA, et al, 2021: A review of Cryptosporidium spp. and their detection in water. Water Sci. Technol. 83, 1:1-25.

Ibrahim, S, Choumane, W, Dayoub, A, 2020: Occurrence and seasonal variations of Giardia in wastewater and river water from Al-Jinderiyah Region in Latakia, Syria. Int. J. Environ. Stud. 77, 3:370-81.

Khairy, AE, El Sebaie, O, Gawad, AA, El Attar, L, 1982: The sanitary condition of rural drinking water in a Nile Delta village. I. Parasitological assessment of 'zir'stored and direct tap water. Epidemiol. Infect. 88, 1:57-61.

LeChevallier, MW, Norton, WD, Lee, RG, 1991: Giardia and Cryptosporidium spp. in filtered drinking water supplies. Appl. Environ. Microbiol. 57, 9:2617-21.

McKee, AM, Cruz, MA, 2021: Microbial and viral indicators of pathogens and human health risks from recreational exposure to waters impaired by fecal contamination. J. Sustain. Water Built. Environ. 7, 2:03121001.

McLauchlin, J, Amar, C, Pedraza-Diaz, S, Nichols, GL, 2000: Molecular Epidemiological analysis of Cryptosporidium spp. in the United Kingdom: Results of genotyping Cryptosporidium spp. in 1,705 fecal samples from humans and 105 fecal samples from livestock animals. J. Clin. Microbiol. 38, 11: 3984-3990.

Medema, GJ, Schets, FM, Ketelaars, H, Boschman, G, 1998: Improved detection and vital staining of Cryptosporidium and Giardia with flow cytometry. Water Sci. Technol. 38, 12:61-5.

Nakada, LYK, Franco, RMB, Fiuza, VRDS, Santos, LUD, Branco, N, et al, 2019: Pre-ozo- nation of source water: Efficacy assessment against Giardia duodenalis cysts \& effects on natural organic matter. Chemosphere 214:764-70.

Nichols, RA, Campbell, BM, Smith, HV, 2006: Molecular fingerprinting of Cryptosporidium oocysts isolated during water monitoring. Appl. Environ. Microbiol. 72, 8:5428-35.

Nichols, RAB, Campbell, BM, Smith, HV, 2003: Identification of Cryptosporidium spp. oocysts in United Kingdom noncarbonated natural mineral waters and drinking waters by using a modified nested PCR-restriction fragment length polymorphism assay. Appl. Environ. Microbiol. 69, 7:4183-9.

Quintero-Betancourt, W, Peele, ER, Rose, JB, 2002: Cryptosporidium parvum and Cyclospora cayetanensis: A review of laboratory methods for detection of these waterborne parasites. J. Microbiol. Meth. 49, 3:209-24.

Rosado-García, FM, Guerrero, M, Karanis, G, Hinojosa, MDC, Karanis, P, 2017: Waterborne protozoa parasites: the Latin American perspective. Int. J. Hyg. Environ. Hlth. 220, 5: 783-98.

Rousseau, A, La Carbona, S, Dumètre, A, Robertson, LJ, Gargala, G, et al, 2018: Assessing viability and infectivity of foodborne and waterborne stages (cysts/oocysts) of Giardia duodenalis, Cryptosporidium spp., and Toxoplasma gondii: A review of methods. Parasite 25: 14.

Sammarro Silva, KJ, Sabogal-Paz, LP, 2020: Analytical challenges and perspectives of asse-ssing viability of Giardia muris cysts and Cryptosporidium parvum oocysts by live/dead simultaneous staining. Environ. Tech. 11:1-10.

Silva, KJS and Sabogal-Paz, LP, 2020: Cryptosporidium spp. and Giardia spp.(oo) cysts as target-organisms in sanitation and environmental monitoring: A review in microscopy-based viability assays. Water Res. 116590.

Ursini, T, Moro, L, Requena-Méndez, A, Bertoli, G, Buonfrate, D, 2020: A review of outbreaks of cryptosporidiosis due to unpasteurized milk. Infection 48:659-63.

Valdez, LM, Dang, HAN, Okhuysen, PC, Chappell, CL, 1997: Flow cytometric detection of Cryptosporidium oocysts in human stool samples. J. Clin. Microbiol. 35, 8:2013-17.

Vesey, G, Hutton, P, Champion, A, Ashbolt, N, Williams, KL, et al, 1994: Application of flow cytometric methods for the routine detection of Cryptosporidium and Giardia in water Cytot- 
ometry. J. Inter. Soci Anal. Cyto. 16, 1:1-6.

Vesey, G, Slade, JS, Byrne, M, Shepherd, K, Dennis, PJ et al, 1993: Routine monitoring of Cryptosporidium oocysts in water using flow cytometry. J. App. Bact. 75, 1:87-90.

Xiao, S, Zhang, Y, Zhao, X, Sun, L, Hu, S,

2018: Presence and molecular characterization of Cryptosporidium and Giardia in recreational lake water in Tianjin, China: A preliminary study. Sci. Rep. 8, 1:1-8.

Yin, YL, Wang, Y, Lai, P, Yao, Q, Li, Y, et al, 2021: Establishment and preliminary application of nanoparticle-assisted PCR assay for detection of Cryptosporidium spp. Parasitol. Res. 2:1-8.

Yoder, J, Roberts, V, Craun, GF, Hill, V, Hicks, L, et al, 2008: Surveillance for waterborne disease \& outbreaks associated with drinking water and water not intended for drinking, United States, 20052006. MMWR Surveil. Summ. 57, 9:39-62.

Zahedi, A, Monis, P, Deere, D, Ryan, U, 2021: Wastewater-based epidemiology-surveillance \& early detection of waterborne pathogens with a focus on SARS-CoV-2, Cryptosporidium and Giardia. Parasitol. Res. 6:1-22

\section{Explanation of figures}

Fig.1: A) Total positive Giardia cysts and Cryptosporidium oocysts in all 64 tanks and tap water samples in different seasons by using different diagnostic techniques. B) Sensitivity, specificity, PPV, NPV, and accuracy of different diagnostic techniques used. C) Total positive samples of Giardia lamblia cysts by nPCR in different seasons in tank and tap water. D) Positive Giardia cyst samples in tank water by different diagnostic techniques in different seasons.

Fig.2: A) Positive Giardia cyst samples in tap water by different diagnostic techniques in different seasons. B) Viability of Giardia cysts by using trypan blue stain and DAPI stain in different seasons in all positive tank and tap water samples. C) Intensity of Giardia cysts/100 $\mu$ by both iodine stain \& IF assay. D) Intensity of Giardia cysts by FC/100 $\mu$.

Fig. 3: A) Sensitivity, specificity, PPV, NPV \& accuracy of different diagnostic techniques to detect Cryptosporidium oocysts in all water samples. B) Total positive samples of Cryptosporidium oocysts by nPCR in different seasons in tank and tap water. C) Positive tank water samples in different seasons by different diagnostic techniques. D) Positive Cryptosporidium oocysts in tap water samples in different seasons by different diagnostic techniques.

Fig. 4: A) Viability of Cryptosporidium oocysts by using conventional trypan blue stain and DAPI stain in different seasons in all positive tank and tap water samples. B) Intensity of Cryptosporidium oocysts/100 $\mu$ by both MZN stain and IF assay. C) Intensity of Cryptosporidium oocysts by FC/100 $\mu$. D) Total positive Cryptosporidium oocysts genotypes in 40 positive tank and tap water samples by nPCR and RFLP.

Fig. 5: A) MZN stained Cryptosporidium oocysts round 4-6 $\mu$ pink in color (acid-fast) against a faint blue-green background (1000x). B) Light trypan blue stained Cryptosporidium oocysts (viable) (1000x). C) Viable Cryptosporidium oocysts (400x). D) Dark stained (dead) Cryptosporidium oocysts by trypan blue (1000x). E) Immunofluorescent bright green Cryptosporidium oocysts (1000x). F) DAPI stained Cryptosporidium oocysts as bright green with shiny apparent nuclei with a faint wall (1000x). G) Entamoeba histolytica cyst distinguished by iodine stain in one sample $(1.56 \%)$. H) Cyclospora cayetanensis oocysts pink (acid-fast) against a faint bluish background in 3 samples (4.7\%) by MZN stain (1000x).

Fig. 6: A) Iodine stained Giardia cysts (1000x). B) MZN stained Giardia cysts (1000x). C) Trypan blue stained (live) Giardia cysts (1000x). D) Dead cysts dark blue by trypan blue stain (400x). D) Immunofluorescence stained Giardia cysts (1000x). E) DAPI stained nuclei of Giardia cysts (1000x).

Fig. 7: A) Negative sample for both Cryptosporidium oocysts and Giardia cysts by flow cytometry. B) Positive sample of Giardia cysts by FC. C) Positive sample of Cryptosporidium oocysts by FC. FC showed all particles of Giardia cysts on a graph as dots called events alongside a histogram. X-axis of histogram represented FITC stained Cryptosporidium oocysts, and Y-axis represents Cy3 stained Giardia cysts, divided into four quadrants. Automatically, device showed each event in histogram by fluorescence intensity. Maximum intensity of FITC stain on $\mathrm{X}$ - axis in lower right quadrant Cryptosporidium oocysts while maximum fluorescence for Cy3 stain on Y-axis in upper left quadrant Giardia cysts.

Fig. 8: A) Agarose gel stained with ethidium bromide corresponding to PCR: Lane L Molecular weight marker (100 bp), Lane 1 Positive control, Lanes 2-6 DNA for products of nPCR of samples targeting TPI gene (530 bp) of Giardia. B) Agarose gel electrophoresis for DNA products; Lane L: 100 bp DNA molecular weight marker. Lanes 1-3: RFLP products after digestion with RsaI endonuclease with C. parvum genotype 2 digestion products at $34,106 \& 410$ bp (34 band very small, faint, but difficult to see). Lanes 4-5: RFLP products after digestion with RsaI endonuclease with $C$. hominis genotype 1 digestion products at $34,106,125 \& 285$ bp ( 34 band very small, faint but difficult to see). Lane 6: Undigested product of COW product at $553 \mathrm{bp}$. Lane 7: Products of nPCR targeting COWP gene of Cryptosporidium at 553bp 

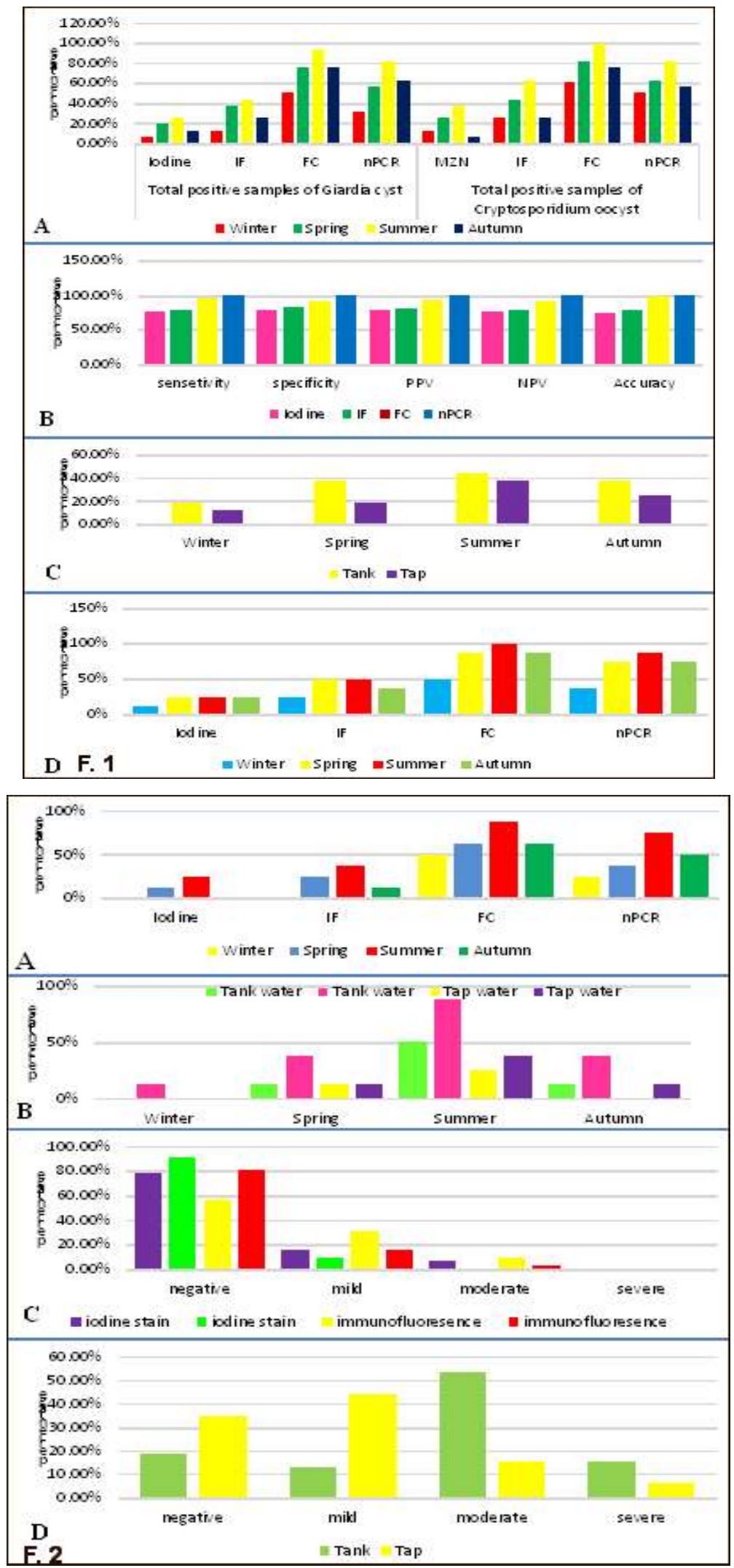

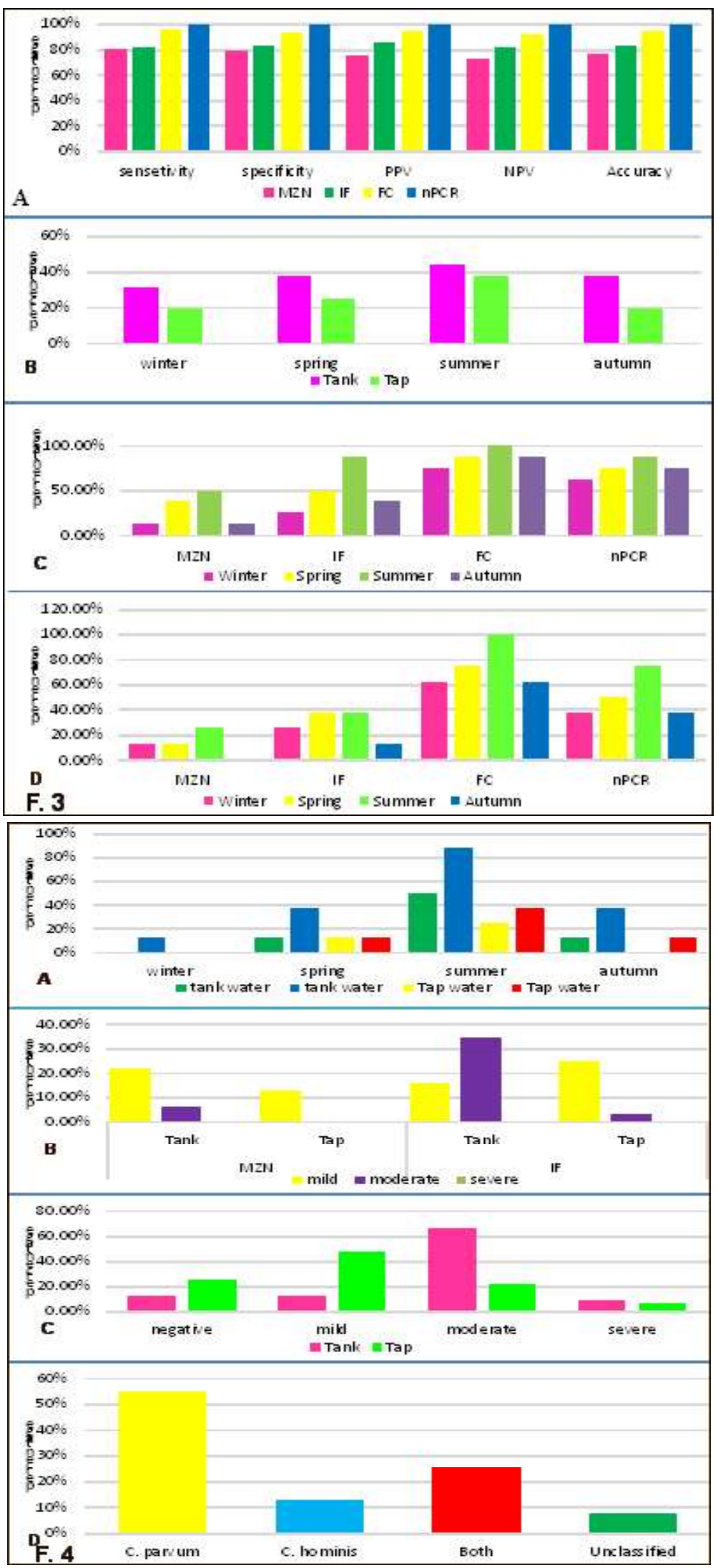

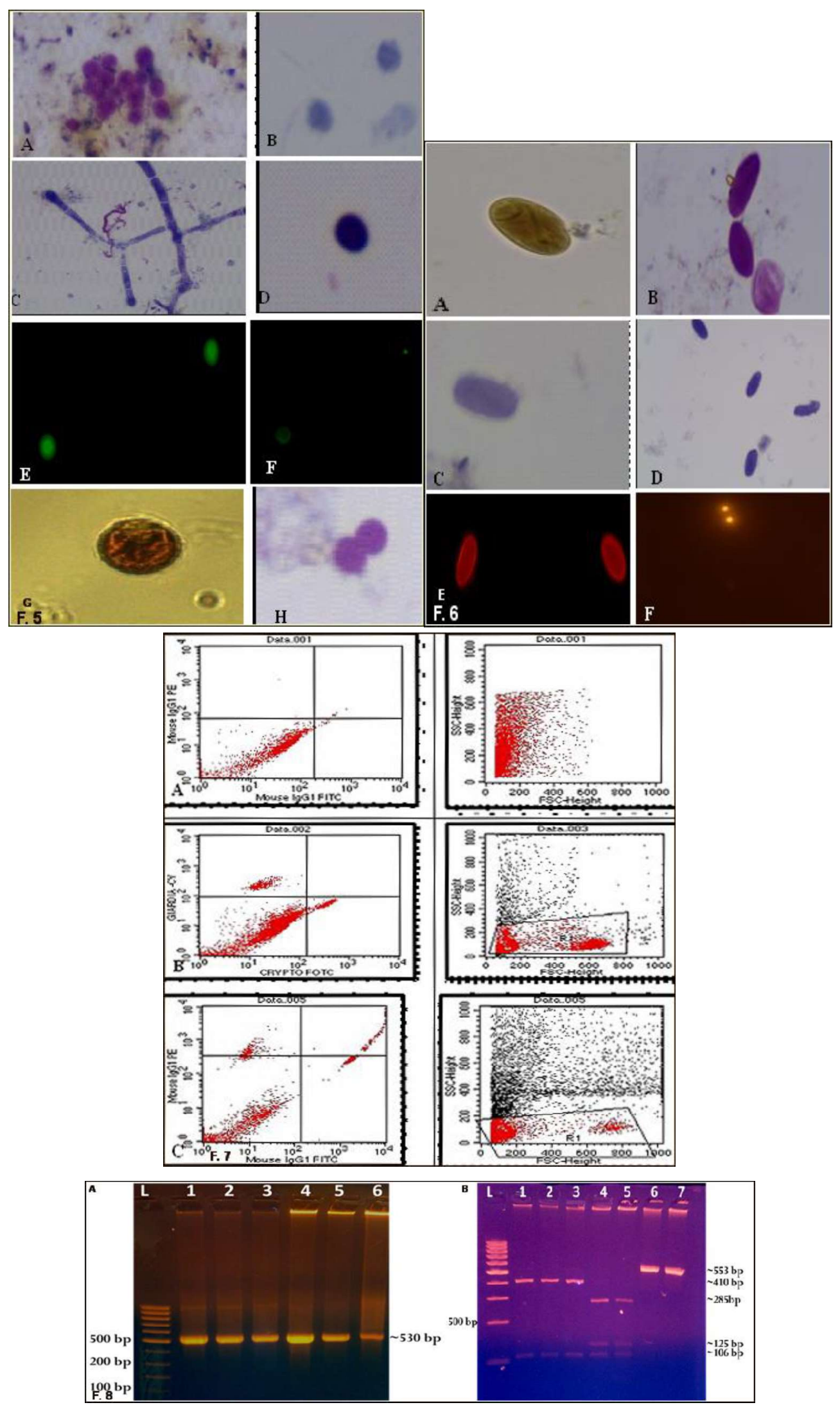